\title{
Habilidades comunicativas y de relación en la educación superior de disciplinas dirigidas al asesoramiento
}

\section{(Communication and Relationship Skills in Higher Education Disciplines Aimed at Counseling)}

\author{
Nieves Valencia-Naranjo \\ $\mathrm{M}^{\mathrm{a}}$ Auxiliadora Robles-Bello \\ Universidad de Jaén (España)
}

DOI: https://doi.org/10.5944/ried.25.1.31327

\section{Cómo referenciar este artículo:}

Valencia-Naranjo, N., y Robles-Bello, M. A. (2022). Habilidades comunicativas y de relación en la educación superior de disciplinas dirigidas al asesoramiento. RIED. Revista Iberoamericana de Educación a Distancia, 25(1), pp. 323-341 preprint). https://doi.org/10.5944/ried.25.1.31327

\section{Resumen}

Las habilidades comunicativas y de relación son relevantes en la actividad académica y profesional en carreras dirigidas a la ayuda a otros. La planificación de actividades formativas dirigidas a la interacción con otros (ej. pares) facilita la adquisición de estas habilidades, especialmente cuando se impulsa mediante la retroalimentación juicios evaluativos más ajustados y la regularización del proceso de aprendizaje. Disponer de instrumentos que faciliten una retroalimentación eficaz contribuye a la planificación de estas tareas formativas, herramientas que deben informar de habilidades que participan en el asesoramiento. Además, facilitan la evaluación eficiente de la adquisición y aprendizaje de amplios grupos de alumnos. Uno de esos instrumentos puede ser el Cuestionario de Habilidades Comunicativas (HABICOM). En este trabajo, se examinaron diversos aspectos metodológicos relacionados con el uso de instrumentos de evaluación de las habilidades comunicativas en situaciones de interacción. Los resultados obtenidos en los objetivos propuestos sugieren que: (1) El análisis factorial confirmatorio indica que la estructura factorial del HABICOM y niveles de fiabilidad y validez son aceptables una vez se eliminan algunos ítems; (2) Estas habilidades auto-percibidas son similares en alumnos de Psicología y Trabajo Social; (3) Existe relación en la percepción de las habilidades comunicativas mostradas durante la interacción en un formato auto-aplicado o hetero-aplicado; (4) La percepción del compañero medida con el HABICOM correlaciona con la percepción de las habilidades de ayuda durante la interacción; (5) El análisis de las habilidades del alumnado mediante video se relaciona con las habilidades identificadas por el compañero. 
Palabras claves: psicología clínica; evaluación; competencia comunicativa; materia transversal; retroalimentación.

\begin{abstract}
Communication and relationship skills are relevant in academic and professional activity in professions aimed at helping others. The planning of training activities aimed at interaction with others (e.g. peers) facilitates the acquisition of these skills, especially when it is promoted through feedback, more accurate evaluative judgments and the regularization of the learning process. Having instruments that facilitate effective feedback contributes to the planning of these training tasks, tools that should inform about skills that participate in counseling. In addition, they facilitate the efficient evaluation of the acquisition and learning in large groups of students. One of these instruments can be the Communication Skills Questionnaire (HABICOM). In this paper, various methodological aspects related to the use of communication and relationship skills assessment instruments in interaction situations were examined. The results obtained in the proposed objectives suggest that: (1) The confirmatory factor analysis indicates that the factorial structure of HABICOM and levels of reliability and validity are acceptable once some items are eliminated; (2) These self-perceived abilities are similar in Psychology and Social Work students; (3) There is a relationship in the perception of the communication skills shown during the interaction in a self-applied or hetero-applied format; (4) The perception of the partner measured with the HABICOM correlates with the perception of the helping skills during the interaction; (5) The analysis of the student's skills through video is related to the skills identified by the partner.
\end{abstract}

Keywords: clinical psychology; evaluation; communicative competence; cross-curricular theme; feedback.

Los modelos, estrategias e intereses formativos han ido evolucionando en las universidades. Uno de esos cambios ha sido reconocer la necesidad de que el alumnado pueda regular su propio proceso de aprendizaje. Panadero et al. (2016, 2018) consideran al modelo de Zimmerman como uno de los modelos más influyentes en el estudio de esta capacidad y que incluye tres fases cíclicas y recursivas: (a) Previsión/preparación con análisis de la tarea, fijación de objetivos e identificación de estrategias adecuadas; (b) Desempeño/ejecución que incluye procesos de autocontrol y autoobservación requeridos para valorar si la ejecución está en línea con los objetivos planteados e introducir los ajustes necesarios y (c) Autorreflexión con análisis sobre la calidad del propio trabajo y posibles causas, impulsando modificaciones en prácticas futuras. Esta capacidad para regular el proceso de aprendizaje puede ser incentivada por estrategias que enfatizan la implicación del estudiante en la auto-evaluación considerado un componente esencial de la autorregulación (Panadero et al., 2019).

Gros Salvat y Cano García (2021) destacan el paralelismo entre el feedback y la autorregularización del aprendizaje. La retroalimentación efectiva es un proceso 
activo que facilita el procesamiento en profundidad de la información transmitida, permite elaborar juicios más sofisticados y la actuación en respuesta al feedback (Carless, 2020). Para ello, el énfasis se sitúa no tanto en la transmisión unidireccional de información (ej. de profesores a estudiantes) como en la actuación conjunta de los participantes en el proceso para mejorar los resultados del aprendizaje (Ibarra-Sáiz y Rodríguez Gómez, 2020).

El uso del feedback en tareas formativas con el objetivo de estimular la capacidad de autorregulación se relaciona con el desarrollo del juicio evaluativo de quien ofrece o recibe esa retroalimentación. Tai et al. (2018) sugieren que la valoración del propio trabajo (o de otros) precisa de la capacidad para comprender qué es un trabajo de calidad y de aplicar esa comprensión durante el proceso evaluativo. Ambos componentes mantienen relaciones recíprocas puesto que el acto de evaluar requiere interactuar con una norma (explícita o implícita) fomentando mediante la reflexión una comprensión cada vez más profunda de ella. Su comprensión, a su vez, influye sobre la toma de decisiones implicadas en el acto de evaluar al tener que justificar esas decisiones en relación con los propios conocimientos, la norma en sí misma y el debate con otras personas (ej. compañeros o tutores). La participación en procesos de feedback es una de las estrategias que incentivan el desarrollo del juicio evaluativo, juicio que, aunque es contextual, se basa en la identificación y aplicación de criterios, capacidades que actúan de forma transversal.

En definitiva, el feedback puede proceder de distintos agentes (ej. profesores o compañeros) con los que interacciona el alumnado (Panadero et al., 2019) e incluso la propia persona (Carless, 2020) y su contribución a la capacidad para regular el aprendizaje está influido por un juicio evaluativo de calidad (Carless, 2020). En este contexto, Villarroel y Bruna (2019) defienden el uso de actividades evaluativas dirigidas al aprendizaje conectadas con las desempeñadas en el campo profesional o personal que generen el suficiente desafío cognitivo para fomentar la construcción del conocimiento y favorezcan el desarrollo de un juicio evaluativo ajustado. El desarrollo de actividades evaluativas con un énfasis en las habilidades comunicativas y de relación en estudiantes cuya actividad profesional se produce en un contexto de interacción puede ser idóneo en el proceso formativo del alumnado.

Las habilidades interpersonales y de comunicación son aplicables en campos profesionales que van desde la psicología y el trabajo social hasta la patología del habla y la medicina (Doucet et al., 2013). La mayoría de los planes de estudio de estos grados recogen la instrucción en habilidades de ayuda interpersonal; esto es, estrategias de comunicación que demuestran la atención, el interés, la comprensión y la capacidad de ayuda. Los indicadores de estas competencias incluyen la escucha efectiva, la decodificación precisa de la comunicación verbal y no verbal de los demás, el reconocimiento de las diferencias culturales, la comunicación hábil con diversas personas y la formulación de preguntas útiles. La relevancia de estas habilidades en Psicología se manifiesta en la propuesta de la Asociación Americana de Psicología (APA) que establece la capacidad para interactuar de manera efectiva 
con los demás como uno de los objetivos que determinan la capacitación de los psicólogos (APA, 2019). Diversos factores relacionados con la relación establecida entre cliente y terapeuta (ej. desarrollo de la alianza terapéutica, empatía, consenso en los objetivos, el grado de colaboración con el cliente y la valoración positiva del cliente) proporcionan una base sólida para el desarrollo de las habilidades de ayuda en estudiantes cuyo campo profesional implica, con frecuencia, la interacción con otros (Kee et al., 2018; Norcross y Lambert, 2018; van der Molen, 2020).

En este campo, las tareas de evaluación de los alumnos suelen dirigirse al nivel de conocimientos mediante pruebas de papel y lápiz o situaciones presentadas mediante vídeo. Otro método general de evaluación es la simulación donde el alumno tiene que interaccionar con otra persona (entrenada o no) y evaluar su ejecución. Las simulaciones presentan buenos índices de validez en referencia a "lo que saben hacer" (Hill et al., 2016). Al examinar el procedimiento de evaluación también es necesario atender a la información proporcionada. Los instrumentos auto-aplicados permiten acceder a la percepción del estudiante sobre las propias habilidades en contextos de interacción. El ajuste entre las creencias de los estudiantes sobre su nivel de habilidad y el grado de habilidad real puede ser bajo y afectar a su ejecución. Por ejemplo, Jaeken et al. (2017) hallaron que los estudiantes tienden a sobreestimar sus habilidades antes de recibir un aprendizaje específico dirigido a la mejora de sus habilidades de interacción mientras que tras esta instrucción suelen infraestimarla. Ambas circunstancias pueden deteriorar el desarrollo de su aprendizaje, y su actuación profesional posterior; en el primer caso, al infrautilizar estrategias que fomenten la comprensión de las circunstancias objeto de la interacción y, en el segundo caso, por las dificultades para adaptar las estrategias de una forma dinámica durante el proceso de interacción (al centrar su atención en ellos mismos) (Jaeken et al., 2017). Así mismo, Hill et al. (2016) indican que la contribución de las habilidades de ayuda en el contexto del asesoramiento también depende de la capacidad del cliente (o persona asesorada) para identificar esas habilidades; esto es, el acceso a la percepción de la persona ayudada sobre las habilidades de comunicación y relación durante la interacción con el alumnado es una de las vías de acceso al nivel de desempeño.

La evaluación de las competencias (conocimiento, habilidades y actitudes) requeridas para desempeñar determinadas funciones (Johnson, 2019) debe incorporar un enfoque multidisciplinario, de métodos múltiples y de información múltiple (Vacha-Haaser et al., 2019). El trabajo que se presenta forma parte de un proyecto docente cuyo objetivo general es generar y valorar un programa de formación en habilidades comunicativas y de relación en estudiantes de grado relacionado con el apoyo/asesoramiento de otras personas; concretamente en Psicología en una fase inicial. Hill (2020) o Ruiz et al. (2016), entre otros, establecen las bases de programas formativos en el desarrollo de habilidades de comunicación e interacción en un entorno de ayuda. Estos programas en el nivel de grado implican, usualmente, interacciones con pares en situaciones predefinidas o reales (aprendizaje 
experiencial) con procesos de retroalimentación usualmente unidireccionales (del profesor/tutor al alumno), centrados en la ejecución del alumno asesor y con limitada posibilidad de práctica individual por el número de alumnos participantes. En el desarrollo de este proyecto docente se utiliza como tarea evaluativa la interacción con pares. Sin embargo, se intenta fomentar su carácter formativo mediante elementos de feedback procedentes del tutor, de los compañeros y del alumnado. Para ello es necesario enfatizar las condiciones que hagan eficaz el feedback y que Henderson et al. (2019) relacionan con la capacidad para ofrecerlo y recibirlo, su diseño y la cultura del entorno en el uso de esta estrategia. Estos autores, entre otros (Carless, 2020; Panadero et al., 2019; Tai et al., 2018; Villarroel y Bruna, 2019), establecen que una de esas condiciones es la especificidad del feedback. En esta dirección, esta fase del trabajo enfatiza la valoración de instrumentos que puedan ser transformados en herramientas útiles durante el proceso de feedback en situaciones de interacción.

Uno delos posibles instrumentos es el Cuestionario de Habilidades Comunicativas (HABICOM) de Hernández-Jorge y de la Rosa (2017). Los aspectos examinados (generar motivación, comunicación no verbal, empatía, expresión emocional, expresión oral, transmisión de información, comunicación abierta y escucha) también atañen a habilidades implicadas en el proceso de terapia y/o asesoramiento. Además, el instrumento es sensible al cambio en las habilidades comunicativas y de relación tras un programa de entrenamiento (Hernández-Jorge y de la Rosa, 2018). Relacionar la información ofrecida sobre la percepción de las habilidades comunicativas de los alumnos, mediante instrumentos como el HABICOM, y el nivel de actuación de los estudiantes en tareas de asesoramiento, facilitaría el acceso a información relevante para impulsar la formación de los estudiantes. Sin embargo, como se comentó previamente, es necesario conocer si la auto-percepción coincide con la percepción de estas habilidades por la persona con la que interacciona o la valoración que realiza la persona asesorada sobre las cualidades de la interacción. Igualmente, es necesario establecer su relación con instrumentos que examinan la calidad de la interacción (ej. SPOM, se describe posteriormente) así como con procedimientos más objetivos (ej. análisis de los videos de la interacción). Estos factores conllevaron el planteamiento de una tarea de evaluación formativa en la que los alumnos interaccionasen con otra persona con el objetivo de ayudar a resolver un problema real y que permitiese el acceso al feedback de la persona asesorada así como del tutor. De forma más concreta, los objetivos en este trabajo y en referencia al HABICOM son:

1. Verificar las propiedades psicométricas del HABICOM.

2. Identificar si la percepción de las habilidades comunicativas en situaciones de interacción difiere entre alumnos de distintos grados y/o niveles del grado.

3. Conocer si existe relación entre la percepción de las propias habilidades comunicativas-relacionales y la de la persona con la que se interacciona. 
4. Examinar desde la perspectiva del compañero de interacción la relación entre las habilidades percibidas en el alumnado y la calidad de la interacción.

5. Definir la relación entre las habilidades percibidas durante la interacción por el compañero y su identificación con métodos más objetivos (análisis de vídeo).

\section{MÉTODO}

\section{Participantes}

Los participantes fueron 65 alumnos de Trabajo Social ( 11 hombres y 54 mujeres); 51 alumnos de $2^{\circ}$ de Psicología (2P) (13 hombres y 38 mujeres) y 75 alumnos de tercero de Psicología (3P) (12 hombres y 63 mujeres) con edades entre los 19 y los $42 \operatorname{años}\left(\bar{X}_{\mathrm{TS}}=21.95, \mathrm{SD}_{\mathrm{TS}}=3.58 ; \bar{X}_{2 \mathrm{P}}=21.43, \mathrm{SD}_{2 \mathrm{P}}=2.16 ; \bar{X}_{3 \mathrm{P}}=22.59, \mathrm{SD}_{3 \mathrm{P}}=2.59\right)$.

\section{Procedimiento}

Se propuso a alumnos de Psicología y Trabajo Social responder al HABICOM y acceder a créditos extras (también disponibles con otras actividades) así como a los resultados obtenidos mediante un informe de feedback. El cuestionario HABICOM se presentó mediante un formulario Google Form utilizando los servicios de la plataforma de la Universidad. El alumnado de tercero de Psicología, además complementaban esta actividad con otra en la que interaccionaban durante un período de aproximadamente 5 minutos con una persona conocida de su entorno social (familiar, amigo, pareja) y cuyo objetivo era escuchar y ayudar a resolver un problema (no íntimo) al que estuviese expuesto el compañero. Esta interacción era grabada en video y compartida con el profesor mediante Google Drive de forma privada. Además, el alumno y el compañero de interacción contestaban al resto de instrumentos. El comité de bioética de la Universidad del primer autor aprobó el trabajo de investigación (MAR.20/15.PRY). El estudio siguió los principios consagrados en la Declaración de Helsinki (Goodyear et al., 2007).

\section{Instrumentos de evaluación}

Cuestionario de Habilidades Comunicativas (HABICOM) (Hernández-Jorge y de la Rosa, 2017). Este cuestionario fue contestado por el alumnado participante e incluye 8 escalas: (1) Generar motivación - generar expectativas positivas mostrando atención y valorando al interlocutor en un clima positivo, cálido y cercano; (2) Comunicación no verbal - ajustar el contacto ocular, la expresión facial y gestual, proximidad y paralenguaje a la interacción; (3) Empatía - entender las emociones y situaciones del otro con una actitud de respeto y tolerancia; (4) Expresión Emocional - dar información sobre sí mismo y expresar las propias necesidades y 
emociones manifestando cercanía; (5) Expresión Oral - vocalizar correctamente con un volumen, modulación y velocidad de habla adecuados; (6) Transmisión de información - ofrecer información de forma comprensible, estructurada y distintiva; (7) Comunicación abierta y auténtica - manifestarse sin engaños, mantener congruencia entre el mensaje verbal y no verbal, mostrar espontaneidad verbal y no verbal y querer transmitir y participar en la comunicación; (8) Recabar información y escuchar - mirar, asentir y no interrumpir, y realizar preguntas. El HABICOM, además, fue puntuado por el compañero de interacción del alumnado de tercero de Psicología. Para ello, los ítems del cuestionario fueron adaptados para referirse a las habilidades observadas en el alumnado. Para ambos participantes (alumnos, compañeros) los ítems se puntuaban en una escala Likert de 5 puntos.

Proceso de la sesión y medidas de resultado (SPOM) (Hill y Kellems, 2002). Este auto-informe de 21 elementos está diseñado para medir las percepciones de la persona asesorada (compañero) sobre las habilidades del terapeuta/asesor aplicadas durante la sesión de interacción. El instrumento consta de tres escalas: (a) Medida de Habilidades de Ayuda (HSM) que examina habilidades relacionadas con la exploración del problema, facilitación de la comprensión de la situación por el cliente e incentivación de la acción; (b) Escala de Relación donde se valora la cualidad de la interacción (ej. confianza en el terapeuta) y (c) Escala de Evaluación de la Sesión (SES) dirigido a la evaluación global de la sesión por la persona asesorada (ej. esta sesión fue útil). Las respuestas se proporcionan en una escala Likert de 5 puntos.

Análisis de vídeo. Se construyó una rúbrica para definir y valorar las habilidades de: (1) Obtención de información a través de preguntas cerradas y abiertas relacionadas con los pensamientos, los sentimientos y las acciones; (2) Habilidades para expresar el proceso de escucha - reflejo, paráfrasis, clarificación síntesis; (3) Habilidades para fomentar el proceso de comprensión - preguntas avanzadas, confrontación/reto, interpretación; (4) Habilidades para incentivar la acción instrucciones, información; (5) Habilidades para fomentar la relación y actuación de la persona asesorada - aprobación y generar un entorno de seguridad e inmediatez y (6) Habilidades no verbales - contacto ocular, volumen y velocidad de habla, apertura corporal, distancia interpersonal, control de gestos no intencionales. Esta rúbrica contenía la descripción de los aspectos básicos de cada habilidad y diversos ejemplos de su aplicación. Los elementos incluidos en la rúbrica fueron discutidos hasta alcanzar un consenso entre los miembros del proyecto.

\section{Análisis de datos}

Se realizó un análisis factorial confirmatorio (CFA) utilizando el método robusto de máxima verosimilitud por la ausencia de normalidad multivariante (coeficiente de Mardia = 22.91). El ajuste del modelo se evalúa mediante el Índice de ajuste comparativo (CFI), Índice de Tucker-Lewis (TLI) -valores iguales o superiores a .95 indican un buen ajuste; la raíz cuadrática media estandarizada residual (SRMR) 
y la raíz cuadrática media del error de aproximación (RMSEA) -los valores por debajo de .06 indican un buen ajuste (Kline, 2015). Este análisis se realizó con el programa R-Studio. Respecto a la confiabilidad de constructo, se calcularon los valores de Fiabilidad compuesta (FC), alfa de Cronbach y omega de McDonald. En términos de validez discriminante se utilizó la proporción HTMT; esto es, la media de las correlaciones entre los indicadores que miden diferentes constructos (correlación HT, Heterotrait-Heteromethod), en relación con el promedio de las correlaciones de indicadores dentro del mismo constructo (correlaciones MT, Monotrait-Heteromethod). Valores inferiores a 0.90 demuestran una adecuada validez discriminante (Henseler et al., 2015).

Para determinar posibles diferencias entre los grupos de alumnos participantes -Psicología (segundo y tercero) y Trabajo Social- en la puntuación total y/o las escalas del HABICOM se aplicaron análisis descriptivos (medias y desviaciones estándar) y de varianza (ANOVA) unidireccional. Así mismo, la posible relación entre las percepciones del alumno y del compañero de interacción en las puntuaciones del HABICOM, entre la valoración del compañero en el HABICOM y su valoración de la sesión de asesoramiento, así como entre su valoración del asesoramiento y el análisis por jueces de la interacción con el alumno, se realiza mediante análisis de correlación de Pearson utilizando el programa SPSS v.24.

\section{RESULTADOS}

Se realizó un análisis factorial confirmatorio (CFA) utilizando el modelamiento de ecuaciones estructurales con las 8 variables latentes establecidas inicialmente en el HABICOM, en un conjunto de datos sin valores perdidos para datos robustos dada la falta de normalidad multivariante. Los resultados del modelo indican un buen ajuste $\left(\mathrm{CFI}_{\mathrm{R}}=0.972 ; \mathrm{TLI}_{\mathrm{R}}=0.968 ; \mathrm{RMSEA}_{\mathrm{R}}=0.041-\mathrm{IC}_{\text {Inferior }}=0.036 ; \mathrm{IC}_{\text {Superior }}=\right.$ 0.046; SRMR = 0.067). Sin embargo, las cargas factoriales sugerían limitada validez convergente, con valores $<0.5$ en los ítems 2, 3 y 4 de la escala Transmisión de Información; ítem 5 dela escala Expresión Oral; ítems 9 y 12 dela escala Comunicación no Verbal; ítem 21 de la escala Comunicación Abierta e ítem 29 de la escala Empatía. Además, no se obtenía validez discriminante entre las escalas Transmisión de Información y Expresión Oral por el método de la varianza compartida (html= 0.91; $>0.8$ ).

Se procedió a la eliminación de los elementos con baja carga factorial. Además, el elemento 1 de la escala Transmisión de Información se anexó a los datos de la escala Expresión Emocional al eliminar los ítems 2, 3 y 4 y quedar infrarrepresentada dicha escala. Los resultados obtenidos en este nuevo modelo siguen ofreciendo un buen ajuste $\left(\mathrm{CFI}_{\mathrm{R}}=0.979 ; \mathrm{TLI}_{\mathrm{R}}=0.975 ; \mathrm{RMSEA}_{\mathrm{R}}=0.040-\mathrm{IC}_{\text {Inferior }}=0.033 ; \mathrm{IC}_{\text {Superior }}=0.047\right.$; $\mathrm{SRMR}=0.063)$ (ver Tabla 1). Así mismo, todos los elementos presentaban una carga factorial superior a 0.5. Los niveles de fiabilidad mediante el alpha de Cronbach se situaban entre 0.635 y 0.791 y los valores omega de MacDonald se encontraban entre 
0.591 y 0.787 (ver Tabla 2). El análisis confirmatorio utilizando una metodología de modelos estructurales, sugiere que la escala HABICOM reducida incluye 26 elementos distribuidos en 7 escalas (Generar Motivación con 6 elementos; Comunicación no Verbal con 3 elementos; Empatía con 3 elementos; Expresión Emocional con 3 elementos; Expresión Oral con 4 elementos; Comunicación Abierta con 4 elementos y Escucha con 3 elementos) muestra un buen ajuste, validez convergente, validez discriminante y valores de fiabilidad aceptables. La Figura 1 representa el modelo de medida obtenido en HABICOM.

\section{Tabla 1}

Resumen de estadísticos descriptivos del modelo de medida del HABICOM

\begin{tabular}{|c|c|c|c|c|c|c|c|}
\hline \multicolumn{5}{|c|}{$\begin{array}{l}\text { Variables latentes e } \\
\text { indicadores: }\end{array}$} & \multicolumn{3}{|c|}{ Varianzas } \\
\hline & Estimador & $\mathbf{P}(>|\mathbf{z}|)$ & $\begin{array}{l}\text { Limite } \\
\text { Inferior }\end{array}$ & $\begin{array}{l}\text { Limite } \\
\text { Superior }\end{array}$ & SD & Estimador & SD \\
\hline \multicolumn{8}{|c|}{ Generar Motivación } \\
\hline $\mathrm{H} 14$ & 1.000 & & 1.000 & 1.000 & 0.679 & 0.342 & 0.539 \\
\hline H15 & 1.025 & 0.000 & 1.229 & 0.554 & 0.702 & 0.315 & 0.508 \\
\hline H16 & 0.862 & 0.000 & 0.673 & 1.051 & 0.646 & 0.304 & 0.583 \\
\hline H17 & 0.841 & 0.000 & 0.570 & 1.113 & 0.508 & 0.593 & 0.742 \\
\hline $\mathrm{H} 25$ & 0.69 & 0.000 & 0.516 & 0.866 & 0.586 & 0.267 & 0.657 \\
\hline $\mathrm{H} 26$ & 0.772 & 0.000 & 0.539 & 1.006 & 0.600 & 0.310 & 0.640 \\
\hline \multicolumn{8}{|c|}{ Comunicación no Verbal } \\
\hline H10 & 1.000 & & 1.000 & 1.000 & 0.580 & 0.581 & 0.663 \\
\hline H11 & 0.850 & 0.000 & 0.610 & 1.089 & 0.551 & 0.489 & 0.697 \\
\hline $\mathrm{H} 13$ & 1.174 & 0.000 & 0.826 & 1.523 & 0.667 & 0.508 & 0.556 \\
\hline \multicolumn{8}{|c|}{ Empatía } \\
\hline $\mathrm{H} 27$ & 1.000 & & 1.000 & 1.000 & 0.644 & 0.191 & 0.585 \\
\hline $\mathrm{H} 28$ & 1.100 & 0.000 & 0.714 & 1.486 & 0.626 & 0.258 & 0.608 \\
\hline H33 & 0.964 & 0.000 & 0.607 & 1.321 & 0.600 & 0.224 & 0.640 \\
\hline \multicolumn{8}{|c|}{ Expresión Emocional } \\
\hline $\mathrm{H} 22$ & 1.000 & & 1.000 & 1.000 & 0.507 & 0.782 & 0.743 \\
\hline $\mathrm{H} 23$ & 1.006 & 0.000 & 0.670 & 1.342 & 0.506 & 0.795 & 0.744 \\
\hline $\mathrm{H} 24$ & 1.035 & 0.000 & 0.662 & 1.408 & 0.810 & 0.124 & 0.345 \\
\hline \multicolumn{8}{|c|}{ Expresión Oral } \\
\hline H6 & 1.000 & & 1.000 & 1.000 & 0.620 & 0.658 & 0.616 \\
\hline $\mathrm{H}_{7}$ & 1.035 & 0.000 & 0.777 & 1.293 & 0.689 & 0.488 & 0.526 \\
\hline H8 & 0.767 & 0.000 & 0.516 & 1.019 & 0.517 & 0.661 & 0.732 \\
\hline H1 & 0.702 & 0.000 & 0.483 & 0.922 & 0.632 & 0.305 & 0.601 \\
\hline
\end{tabular}




\begin{tabular}{|c|c|c|c|c|c|c|c|}
\hline \multicolumn{5}{|c|}{$\begin{array}{l}\text { Variables latentes e } \\
\text { indicadores: }\end{array}$} & \multicolumn{3}{|c|}{ Varianzas } \\
\hline & Estimador & $P(>|z|)$ & $\begin{array}{l}\text { Limite } \\
\text { Inferior }\end{array}$ & $\begin{array}{l}\text { Limite } \\
\text { Superior }\end{array}$ & SD & Estimador & SD \\
\hline \multicolumn{8}{|c|}{ Escucha } \\
\hline $\mathrm{H} 18$ & 1.000 & & 1.000 & 1.000 & 0.521 & 0.605 & 0.728 \\
\hline H19 & 1.173 & 0.000 & 0.734 & 1.612 & 0.643 & 0.441 & 0.587 \\
\hline $\mathrm{H} 2 \mathrm{O}$ & 1.319 & 0.000 & 0.878 & 1.759 & 0.730 & 0.344 & 0.467 \\
\hline \multicolumn{8}{|c|}{ Comunicación Abierta } \\
\hline $\mathrm{H}_{3} \mathrm{O}$ & 1.000 & & 1.000 & 1.000 & & 0.461 & 0.688 \\
\hline $\mathrm{H} 31$ & 1.072 & 0.000 & 0.785 & 1.360 & 0.681 & 0.278 & 0.537 \\
\hline $\mathrm{H}_{32}$ & 1.163 & 0.000 & 0.764 & 1.562 & 0.580 & 0.558 & 0.664 \\
\hline $\mathrm{H} 34$ & 1.177 & 0.000 & 0.770 & 1.584 & 0.646 & 0.403 & 0.582 \\
\hline
\end{tabular}

Tabla 2

Índices de fiabilidad en la escala HABICOM

\begin{tabular}{|c|c|c|c|c|c|c|c|}
\hline & GenMot & ComNVer & Emp & ExpEmo & ExpOral & Esc & CA \\
\hline \multicolumn{8}{|l|}{ HTMT } \\
\hline $\begin{array}{l}\text { Generar } \\
\text { Motivación }\end{array}$ & 1 & & & & & & \\
\hline $\begin{array}{l}\text { Comunicación } \\
\text { no Verbal }\end{array}$ & 0.800 & 1.000 & & & & & \\
\hline Empatía & 0.830 & 0.643 & 1.000 & & & & \\
\hline $\begin{array}{l}\text { Expresión } \\
\text { Emocional }\end{array}$ & 0.729 & 0.648 & 0.485 & 1.000 & & & \\
\hline Expresión Oral & 0.834 & 0.874 & 0.558 & 0.671 & 1.000 & & \\
\hline Escucha & 0.716 & 0.544 & 0.690 & 0.528 & 0.456 & 1.000 & \\
\hline $\begin{array}{l}\text { Comunicación } \\
\text { Abierta }\end{array}$ & 0.638 & 0.791 & 0.709 & 0.727 & 0.633 & 0.401 & 1.000 \\
\hline Alpha & 0.791 & 0.635 & 0.675 & 0.645 & 0.699 & 0.658 & 0.698 \\
\hline Omega & 0.787 & 0.631 & 0.655 & 0.591 & 0.705 & 0.664 & 0.705 \\
\hline $\begin{array}{l}\text { Fiabilidad } \\
\text { compuesta } \\
(\mathrm{FC}): 0.9832\end{array}$ & & & & & & & \\
\hline
\end{tabular}




\section{Figura 1}

Modelo de medida para el HABICOM. Las elipses representan las variables latentes y los rectángulos representan las variables medidas. Las varianzas explicadas se indican encima de las flechas pequeñas. Las flechas largas recogen la relación entre las variables latentes

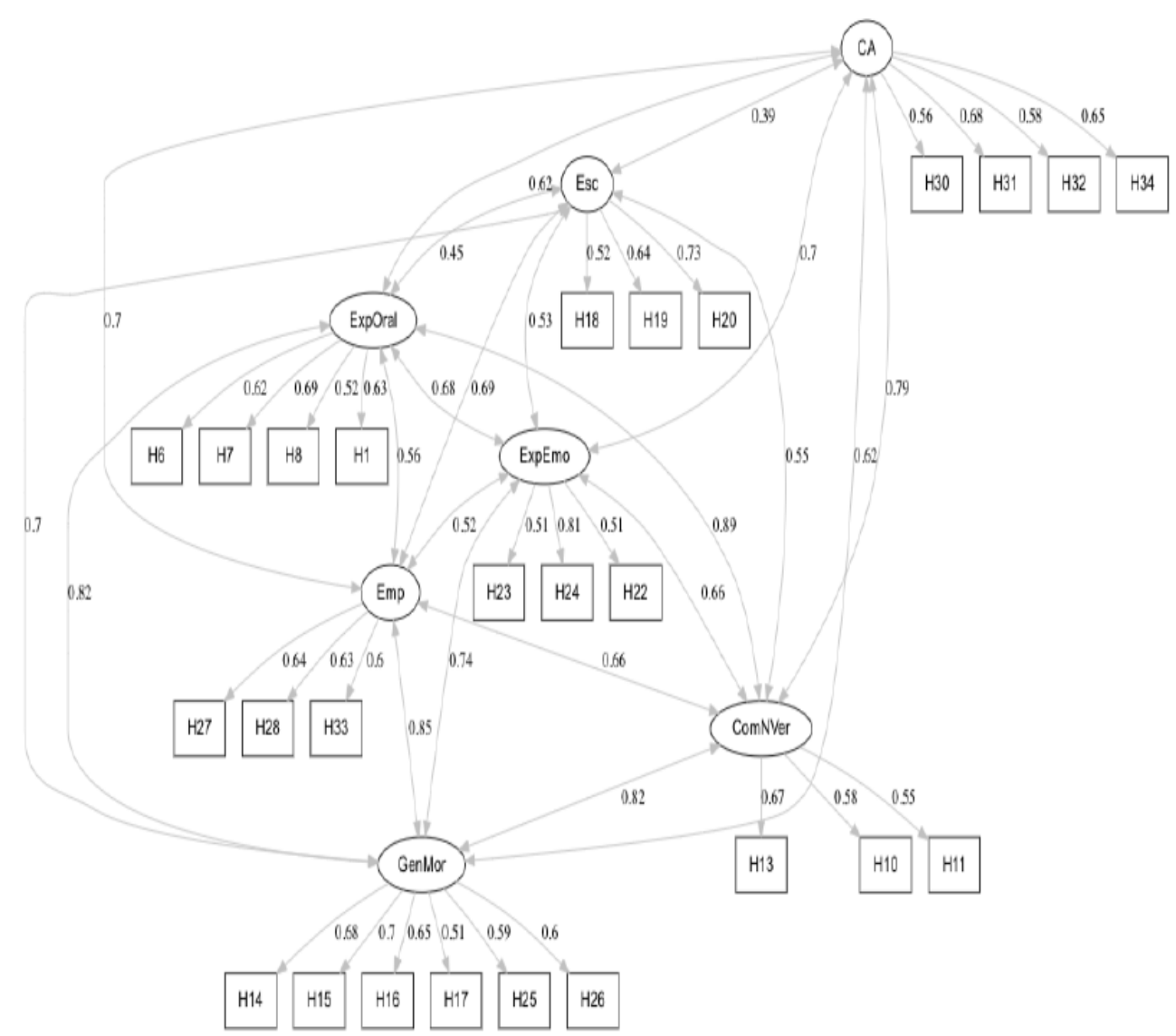

Nota: "GenMot", Generar Motivación; "ComNVer", Comunicación no Verbal; "Emp", Empatía; “ExpEmo”, Expresión Emocional; “ExpOral”, Expresión Oral; “Esc”, Escucha; "CA", Comunicación Abierta.

En relación con el segundo objetivo sobre posibles diferencias entre los alumnos según grado y curso, los resultados sugieren que existen diferencias en la puntuación de Total $\left(\mathrm{F}_{(2,188)}=4.051, \mathrm{p}<0.05\right)$, Generar Motivación $\left(\mathrm{F}_{(2,188)}=5.521, \mathrm{p}<0.05\right) \mathrm{y}$ Comunicación no Verbal $\left(\mathrm{F}_{(2,188)}=4.298, \mathrm{p}<0.05\right)$, con valores medios superiores en los alumnos de $3^{\circ}$ de Psicología vs Trabajo Social. Las diferencias eran marginales en 
Comunicación Abierta $\left(\mathrm{F}_{(2,188)}=2.510, \mathrm{p}=0.084\right)$. Además, en la subescala Generar Motivación ( $\mathrm{p}=0.07)$, estas diferencias eran marginales entre los alumnos de $2^{\circ} \mathrm{de}$ Psicología y de Trabajo social (ver Tabla 3).

\section{Tabla 3}

Medias, desviaciones típicas, valor de $F$ y diferencias entre los grupos por diplomatura y curso en las escalas del HABICOM

\begin{tabular}{|c|c|c|c|c|c|}
\hline & $\bar{X}^{\mathbf{3}}$ (SD) & $\bar{X}_{\text {(SD) }}^{\mathbf{2 P}}$ & $\bar{X} \stackrel{\text { TS }}{\text { (SD) }}$ & $\mathbf{F}_{(2,188)}$ & $\begin{array}{l}\text { Diferencias } \\
\text { de. Grupos }\end{array}$ \\
\hline Total & $109.96(11.11)$ & $106.61(12.13)$ & $104.46(11.52)$ & $4.051^{*}$ & $3 \mathrm{P} / \mathrm{TS}$ \\
\hline Generar Motivación & $26.05(2.87)$ & $25.69(3.18)$ & $24.35(3.33)$ & $5 \cdot 521^{* *}$ & $3 \mathrm{P} / \mathrm{TS}$ \\
\hline $\begin{array}{l}\text { Comunicación no } \\
\text { Verbal }\end{array}$ & $12.47(1.99)$ & $12.14(2.03)$ & 11.46 (2.09) & $4.298^{*}$ & $3 \mathrm{P} / \mathrm{TS}$ \\
\hline Empatía & $13.92(1.18)$ & $13.39(1.67)$ & $13.61(1.40)$ & 2.245 & \\
\hline $\begin{array}{l}\text { Expresión } \\
\text { Emocional }\end{array}$ & $11.81(2.03)$ & $11.45(2.20)$ & $11.23(2.14)$ & 1.355 & \\
\hline Expresión Oral & $16.05(2.50)$ & $15.74(2.53)$ & $15.12(2.91)$ & 2.169 & \\
\hline $\begin{array}{l}\text { Comunicación } \\
\text { Abierta }\end{array}$ & $17.19(2.35)$ & $16.27(2.45)$ & $16.55(2.33)$ & 2.510 & \\
\hline Escucha & $12.47(1.88)$ & $11.92(2.23)$ & $12.12(2.03)$ & 1.172 & \\
\hline
\end{tabular}

Para los objetivos 3, 4 y 5, los participantes fueron 40 alumnos de $3^{\circ}$ de Psicología quienes interaccionaron con una persona de su entorno social inmediato y en relación con un problema mostrado por esa persona. Esta interacción era grabada en video. La correlación bilateral de Pearson se utilizó para examinar la relación entre la autopercepción de los alumnos y la percepción del compañero de interacción mediante el HABICOM (objetivo 3); la relación entre la valoración realizada por el compañero de las habilidades medidas con el HABICOM y de la sesión de interacción mediante el SPOM (objetivo 4) y la relación entre la cualidad de la interacción (SPOM) establecida por el compañero y las habilidades identificadas en el estudiante mediante el análisis del video de la interacción (objetivo 5). El acuerdo inter-jueces, obtenido a través del coeficiente kappa de Cohen, para las distintas habilidades evaluadas a partir del video se situó entre 0.60 y 0.80 .

La correlación entre la valoración de las habilidades examinadas con el HABICOM de forma auto o hetero aplicada es significativa en Total ( $\mathrm{r}_{\mathrm{xy}}=0.530$; $\left.\mathrm{p}<0.001\right)$; Generar Motivación $\left(\mathrm{r}_{\mathrm{xy}}=0.381 ; \mathrm{p}<0.05\right)$; Comunicación no Verbal $\left(\mathrm{r}_{\mathrm{xy}}=0.419 ; \mathrm{p}\right.$ $<0.05)$; Expresión Oral $\left(r_{x y}=0.522 ; p<0.001\right)$ y Escucha $\left(r_{x y}=0.448 ; p<0.05\right)$ y de forma marginal en Empatía $\left(r_{x y}=0.269 ; p=0.093\right)$ y Expresión Emocional $\left(r_{x y}=\right.$ 
0.303; $\mathrm{p}=$ 0.057), sin alcanzar este criterio estadístico en Comunicación Abierta $\left(\mathrm{r}_{\mathrm{xy}}=\right.$ $0.258 ; \mathrm{p}=0.109$ ).

Así mismo, las diferencias de medias (prueba t) en las percepciones de los dos miembros de la interacción indicaban que la valoración de los compañeros eran superiores en Total $\left(\mathrm{t}_{(39)}=3.119 ; \mathrm{p}<0.05\right)$, Expresión Emocional $\left(\mathrm{t}_{(39)}=2.257 ; \mathrm{p}<\right.$ o.05); Expresión Oral $\left(\mathrm{t}_{(39)}=3.539 ; \mathrm{p}<0.001\right.$; Comunicación Abierta $\left(\mathrm{t}_{(39)}=3.312\right.$; $\mathrm{p}<0.05)$ y Escucha $\left(\mathrm{t}_{(39)}=2.525 ; \mathrm{p}<0.05\right)$ con diferencias marginales en Generar Motivación $\left(\mathrm{t}_{(39)}=2.018 ; \mathrm{p}=0.051\right)$ y sin diferencias en Comunicación no Verbal $\left(\mathrm{t}_{(39)}=1.406 ; \mathrm{p}=0.168\right)$ y Empatía $\left(\mathrm{t}_{(39)}=0.808 ; \mathrm{p}=0.424\right)($ ver Tabla 4$)$.

\section{Tabla 4}

Medias, desviaciones típicas, valor de $t$ y diferencias entre los miembros de la interacción (compañero, alumno) en las escalas del HABICOM

\begin{tabular}{|c|c|c|c|c|}
\hline & $\mathbf{R}_{\mathrm{xy}}$ & $\begin{array}{c}\text { Compañero } \\
X \text { (SD) }\end{array}$ & $\frac{\text { Alumno }}{X}$ (SD) & t (39) \\
\hline Total & $.530^{* *}$ & $114.35(13.88)$ & $108.40(10.02)$ & $3.119^{*}$ \\
\hline Generar Motivación & $.381^{*}$ & $26.72(3.36)$ & $25.62(2.77)$ & 2.018 \\
\hline Comunicac. No Verbal & $.419^{*}$ & $12.80(2.05)$ & $12.32(1.88)$ & 1.406 \\
\hline Empatía & .269 & $13.58(1.60)$ & $13.80(1.28)$ & .808 \\
\hline Expresión Emocional & .303 & $12.47(2.17)$ & $11.60(1.97)$ & $2.257^{*}$ \\
\hline Expresión Oral & $.522^{* *}$ & $17.28(2.87)$ & $15.82(2.34)$ & $3.539^{* *}$ \\
\hline Comunicación Abierta & .258 & $17.19(2.35)$ & $16.27(2.45)$ & $3 \cdot 312^{*}$ \\
\hline Escucha & $.448^{* *}$ & $13.02(1.86)$ & $12.25(1.83)$ & $2.525^{*}$ \\
\hline $\begin{array}{l}{ }^{*} \mathrm{p}<.05 ;{ }^{* *} \mathrm{p}<.001 \\
\mathrm{r}_{\mathrm{xy}:} \text { correlación bilatera }\end{array}$ & & & & \\
\hline
\end{tabular}

La puntuación total en el HABICOM del compañero correlacionaba significativamente con la valoración que hacía esa persona sobre el uso de técnicas comunicativas y de relación referidas a la exploración del problema $\left(\mathrm{r}_{\mathrm{xy}}=0.479 ; \mathrm{p}\right.$ $<0.001)$, facilitación de la comprensión $\left(\mathrm{r}_{\mathrm{xy}}=0.754 ; \mathrm{p}<\mathrm{0.001}\right)$ e incentivación de acciones $\left(\mathrm{r}_{\mathrm{xy}}=0.357 ; \mathrm{p}<0.05\right)$ así como cuando valora la utilidad de la sesión $\left(\mathrm{r}_{\mathrm{xy}}=\right.$ $0.442 ; \mathrm{p}<0.05)$. Esta relación no era significativa al examinar la relación establecida con el alumno $\left(r_{x y}=0.231 ; p=0.151\right)$.

En cuanto a las habilidades de interacción y comunicación a través del análisis del video de la interacción, los resultados muestran que existe una relación significativa entre el número total de habilidades puestas en práctica por los alumnos durante la interacción y la valoración que realiza el compañero de interacción en el SPOM $\left(\mathrm{r}_{\mathrm{xy} \_ \text {Exploración. }}=0.314 ; \mathrm{p}<0.05 ; \mathrm{r}_{\mathrm{xy} \_ \text {Comprensión }}=0.380 ; \mathrm{p}<0.05 ; \mathrm{r}_{\mathrm{xy} \text { _Acción }}=0.320 ; \mathrm{p}<0.05\right.$; $\mathrm{r}_{\mathrm{xy} \_ \text {Relación }}=0.330 ; \mathrm{p}<0.05$ ) a excepción de la escala de evaluación de la utilidad de la interacción por el compañero $r_{\mathrm{xy} \_ \text {Evaluación }}=0.165 ; \mathrm{p}=0.309$ ). 


\section{DISCUSIÓN}

Los datos obtenidos sugieren que el HABICOM reducido puede ser utilizado para valorar las habilidades comunicativas y de interacción en alumnos que cursan estudios relacionados con la ayuda a otras personas. Además, el instrumento puede aplicarse accediendo a las evaluaciones dela persona con la que interacciona el alumno (hetero-aplicado). Atendiendo a la percepción del compañero de interacción, existe correspondencia entre las habilidades del alumno en el HABICOM y las estrategias aplicadas durante la interacción así como entre estas habilidades y la identificación de las habilidades utilizando el análisis de video. Estos resultados sugieren, en general, que los instrumentos seleccionados en esta fase inicial del proyecto pueden ser utilizados en la planificación de sistemas de feedback procedentes de distintas fuentes (propia, compañeros/pares, tutores/profesores) en situaciones de interacción.

De forma más específica, y en relación con el objetivo 1, el análisis factorial confirmatorio muestra que la versión reducida del HABICOM (Hernández-Jorge y de la Rosa, 2017) de 26 elementos distribuidos en 7 subescalas (se elimina la subescala Transferencia de Información) presenta niveles de validez convergente y discriminante superiores a las del instrumento original y alcanza niveles de fiabilidad (alpha de Cronbach y omega) aceptables en las distintas subescalas.

Kuntze et al. (2018) o Van der Molen (2020) diferencian entre habilidades de comunicación y relación dirigidas a la ayuda básicas (ej. empatía, escucha y atención a la expresión oral y emocional) y avanzadas (ej. generar motivación, control de aspectos no verbales, directividad, confrontación, empatía avanzada, etc). Los datos obtenidos utilizando la escala HABICOM reducida apoyan esta distinción (objetivo 2). La percepción de las propias habilidades comunicativas básicas, objetivo de formación en los primeros cursos de los grados, no difiere entre los alumnos; sin embargo, las habilidades más avanzadas se presentan entre los alumnos de tercero de Psicología.

El HABICOM puede administrarse en su formato original auto-aplicado pero también es fácil la adaptación a un formato hetero-aplicado obteniéndose buenos niveles de relación entre ambas modalidades como sugieren la alta correlación de las puntuaciones. Sin embargo, y en relación al objetivo 3 de este trabajo, los alumnos tienden a considerar que sus habilidades implicadas en la ayuda se encuentran menos desarrolladas (vs sus compañeros de interacción). Similares distorsiones fueron obtenidas por Jaeken et al. (2017). El feedback asociado a una única interacción no ha sido suficiente para facilitar un juicio evaluativo más ajustado en el alumnado. Además, probablemente estas condiciones limitan la facilitación del aprendizaje autorregulado sugerido por autores como Panadero et al. (2019) o Gros Salvat y Cano García (2021) quienes implican al feedback en la optimización de competencias y habilidades en las distintas fases (planificación, desarrollo y reflexión) del proceso de regularización del aprendizaje. 
El feedback procedente de distintas fuentes externas de información aumenta la eficacia de esta herramienta en la adquisición de aprendizajes y fomenta el autofeedback (Carless, 2020). Una de esas fuentes de feedback es el propio tutor. Cobb et al. (2020) destacan que en alumnos de postgrado, la percepción del supervisor que guía el proceso de aprendizaje del alumnado, y, especialmente, la forma en la que comunica esa percepción, influye en las creencias de autoeficacia del alumno. Estos autores destacan como posibles estrategias a utilizar por el supervisor el implicar al alumnado en la actividad a desarrollar, expresar con frecuencia sus creencias sobre fortalezas y áreas de crecimiento/desarrollo detectadas en el alumnado y apoyarles en aquellas tareas que resulten más complejas mediante la comunicación de la capacidad/dominio del alumno en esa habilidad o intentar persuadirlo verbalmente alentándole (y apoyándole) en el logro de esa habilidad.

Otra posible fuente de retroalimentación son los pares. Diversos autores (Carless, 2020; Gros Salvat y Cano García, 2021; Panadero et al., 2019) destacan el papel de esta estrategia de evaluación en el aprendizaje auto-regulado. En este trabajo, la relación significativa entre las puntuaciones globales del HABICOM administrado con un formato auto/hetero aplicado (objetivo3), junto a la correlación entre la percepción del compañero de interacción de las habilidades en el alumno y las habilidades que utiliza durante la interacción de ayuda (medidas mediante el SPOM) (objetivo 4) así como de estas últimas con los datos extraídos del análisis del video por jueces (objetivo 5); sugieren que tareas de interacción centradas en la resolución de un problema real e instrumentos como los utilizados en este trabajo pueden servir de base para la construcción de tareas formativas que utilizan procesos de feedback.

En este sentido, un aspecto relevante es la coincidencia entre las valoraciones subjetivas del compañero en el HABICOM y el SPOM. Sería comprensible que vínculos afectivos más estrechos con el alumnado pudiesen fomentar percepciones positivamente sesgadas de sus habilidades (ej. las interacciones se realizaron con personas de su entorno próximo). Sin embargo, el compañero de interacción es capaz de valorar de forma ajustada las habilidades del alumnado durante la interacción como se infiere de la correspondencia entre la percepción del compañero y las habilidades identificadas mediante el análisis del video, procedimiento considerado más objetivo. Esta buena correlación con las habilidades percibidas en el alumno por el compañero se obtiene en exploración, facilitación de la comprensión, incentivación de la actuación y establecimiento de la relación, pese al corto tiempo de interacción entre el alumno (aproximadamente 5 minutos) e inferior al informado en otros trabajos (ej. Bonacquisti y McElwaine, 2019). Otro aspecto destacable es que el SPOM posibilita acceder a la valoración de la persona ayudada, información que contribuye a mejorar la calidad general del asesoramiento (Hill, 2020; Hill et al., 2008; Vacha-Haase et al., 2019) y no disponible mediante el análisis de vídeo. Estos resultados pueden estar influidos por el conocimiento previo entre participantes, planteando la necesidad de verificar si se mantienen en contextos donde es necesario establecer la relación de trabajo. 
En esta fase del proyecto se han utilizado servicios tecnológicos disponibles en la universidad, especialmente para el intercambio de información (ej. subida de videos, rellenar los cuestionarios, proporcionar el feedback al alumnado) y su análisis. Estas condiciones en el desarrollo de la tarea evaluativa y obtención de la información fueron forzadas por una situación de pandemia y restricciones importantes en la movilidad, ofreciendo una ayuda inestimable en el mantenimiento de los procesos formativos. Pese a eso, la revisión de Gros Salvat y Cano García (2021) proporciona sugerencias que amplían el potencial de estos servicios tecnológicos en la planificación de sistemas de feedback que cumplan, al menos, la condición de estar centrados en la interacción y buscar procesos de feedback orientados a la acción. Serán herramientas que formen parte de actuaciones futuras del equipo.

El potencial de estos servicios tecnológicos y las sugerencias de diversos autores (Carless, 2020; Gros Salvat y Cano García, 2021; Panadero et al., 2019; Tai et al., 2018, entre otros) incentivan la planificación y desarrollo de tareas evaluativas formativas en las que se delimiten estándares de calidad así como los indicadores para alcanzar esos estándares. En cuanto a las habilidades de comunicación e interacción, los instrumentos utilizados en este trabajo ayudan al desarrollo de esos estándares e indicadores. Así mismo, facilitan la planificación de situaciones de interacción en un amplio número de alumnos y de sistemas de feedback que fomenten juicios evaluativos más ajustados (ej. mediante ejemplos, valoración por pares incluyendo comentarios) y aumenten el grado de control sobre el aprendizaje. Para ello, este feedback debe ser comprendido por el alumno y motivar una transformación de la información en elementos concretos que guíen la ejecución futura del alumno. Todos estos elementos deben estar presentes en un entorno que ofrezca distintas oportunidades de interacción (y feedback) permitiendo un progreso mantenido en las competencias que se adquieren. En este sentido, Goodie et al., (2021) destacan el aumento fiabilidad de los procedimientos de evaluación asociados a la ejecución de los alumnos mediante rúbrica. Estas modificaciones están siendo el objetivo de la nueva fase del proyecto docente.

\section{Conflicto de intereses}

Los autores no muestran conflicto de intereses.

\section{Fuentes de financiación}

Este trabajo recibió financiación a través del proyecto PIMED23_20192.

\section{REFERENCIAS}

American Psychological Association. (2019). Standards of accreditation for health 
org/ed/accreditation/about/policies/ standards-of-accreditation.pdf

Bonacquisti, A., y McElwaine, P. (2019). An Experiential Approach to Teaching Counseling Skills: Instructional Modules for Undergraduate and Graduate Students in Psychology. Society for the Teaching of Psychology (STP). https:// teachpsych.org/resources/Documents/ otrp/resources/STP\%20Teaching\%20 Resource\%20-\%20Counseling\%20 Skills\%20-\%20Final.pdf

Carless, D. (2020). From teacher transmission of information to student feedback literacy: Activating the learner role in feedback processes. Active Learning in Higher Education. https:// doi.org/10.1177/1469787420945845

Cobb, C. L., Zamboanga, B. L., Xie, D., Schwartz, S. J., Martinez, C. R., y Skaggs, S. (2020). Associations among the advisory working alliance and research self-efficacy within a relational-efficacy framework. Journal of Counseling Psychology, 67(3), 361-370. https://doi. org/10.1037/couo000389

Doucet, S., Buchanan, J., Cole, T., y McCoy, C. (2013). A team approach to an undergraduatee interprofessional communication course. Journal of Interprofessional Care, 27(3), 272-273. https://doi.org/10.3109/13561820.2012. 743978

Goodie, J. L, Bennion, L. D., Schvey, N. A., Riggs, D. S., Montgomery, M., y Dorsey, R. M. (2021). Development_and implementation of an objective structured clinical examination for evaluating clinical psychology graduate students. Training and Education in Professional Psychology. https://doi.org/10.1037/ tepoo00356

Goodyear M. D., Krleza-Jeric K., y Lemmens T. (2007). The Declaration of Helsinki. BMJ, 335, 624-625. https://doi. org/10.1136/bmj.39339.610000.BE
Gros Salvat, B., y Cano García, E. (2021). Procesos de feedback para fomentar la autorregulación con soporte tecnológico en la educación superior: Revisión sistemática. RIED. Revista Iberoamericana de Educación a Distancia, 24(2), 107-125. https://doi. org/10.5944/ried.24.2.28886

Henderson, M., Phillips, M., Ryan, T., Boud, D., Dawson, P., Molloy, E., y Mahoney, P. (2019). Conditions that enable effective feedback. Higher Education Research \& Development, 38(7), 1401-1416. https:// doi.org/10.1080/07294360.2019.165780 7

Henseler, J., Ringle, C. M., y Sarstedt, M. (2015). A new criterion for assessing discriminant validity in variance-based structural equation modelling. Journal of the Academy of Marketing Science, 43(1), 115-135. https://doi.org/10.1007/s11747014-0403-8

Hernández-Jorge, C., y de la Rosa, C. M (2017). Habilidades comunicativas en estudiantes de carreras de apoyo frente a estudiantes de otras carreras. Apuntes de Psicología, 35, 93-104. http://www. apuntesdepsicologia.es/index.php/ revista/article/view/663

Hernández-Jorge, C., y de la Rosa, C. M. (2018). Percepción de mejora de las habilidades comunicativas en estudiantes universitarios. Revista de la Educación Superior, 47(186), 119-135. https://doi. org/10.36857/resu.2018.186.351

Hill, C. E. (2020). Helping skills: Facilitating Exploration, Insight, and Action (5th ed.). American Psychological Association. https://doi.org/10.1037/0000147-000

Hill, C. E., Anderson, T., Kline, K., McClintock, A., Cranston, S., McCarrick, S., Petrarca, A., Himawan, L., PérezRojas, A. E., Bhatia, A., Gupta, S., y Gregor, M. (2016). Helping skills training for undergraduate students: Who should we select and train? IThe Counseling 
Psychologist, 44, 50-77. https://doi. org/10.1177/0011000015613142

Hill, C. E., y Kellems, I. S. (2002). Development and use of the Helping Skills Measure to assess client perceptions of the effects of training and helping skills in sessions. Journal of Counseling Psychology, 49(2), 264-272. https://doi. org/10.1037/0022-0167.49.2.264

Hill, C. E., Roffman, M., Stahl, J., Friedman, S., Hummel, A., y Wallace, C. (2008). Helping skills training for undergraduates: Outcomes and predictions of outcomes. Journal of Counseling Psychology, 55(3), 359-370. https://doi.org/10.1037/00220167.55.3.359

Ibarra-Sáiz, M. S., y Rodríguez-Gómez, G. (2020). Aprendiendo a evaluar para aprender en la educación superior. Revista Iberoamericana de Evaluación Educativa, 13(1), 5-8. https://doi. org/10.15366/riee2020.13.1.005

Jaeken, M., Zech, E., Brison, C., Verhofstadt, L. L., Van Broeck, N., y Mikolajczak, M. (2017). Helpers' self-assessment biases before and after helping skills training. Frontiers in psychology, 8, 1377. https:// doi.org/10.3389/fpsyg.2017.01377

Johnson, E. A. (2019). Recommendations to enhance psychotherapy supervision in psychology. Canadian Psychology/ Psychologie Canadienne, 6o(4), 290-301. https://doi.org/10.1037/capooo0188

Kee, J., Khoo, H. S., Lim, I., y Koh, M. Y. H. (2018). Communication skills in patientdoctor interactions: Learning from patient complaints. Health Professions Education, 4(2), 97-106. https://doi. org/10.1016/j.hpe.2017.03.006

Kline, R. B. (2015). Principles and Practice of Structural Equation Modeling. Guilford Press.

Kuntze, J., van der Molen, H. T., y Born, M. (2018). Mastery of communication skills. Does intelligence matter? Health Professions Education, 4, 9. https://doi. org/10.1016/j.hpe.2016.08.002
Norcross, J. C., y Lambert, M. J. (2018). Psychotherapy relationships that work III. Psychotherapy, 55(4), 303-315. https:// doi.org/10.1037/pst0000193

Panadero, E., Andrade, H., y Brookhart, S. (2018). Fusing self-regulated learning and formative assessment: A roadmap of where we are, how we got here, and where we are going. The Australian Educational Researcher, 45, 13-31. https://doi. org/10.1007/s13384-018-0258-y

Panadero, E., Broadbent, J., Boud, D., y Lodge, J. M. (2019). Using formative assessment to influence self- and coregulated learning: The role of evaluative judgement. European Journal of Psychology of Education, 34(3), 535-557. https://doi.org/10.1007/s10212-0180407-8

Panadero E., Jonsson A., y Strijbos J. W. (2016) Scaffolding self-regulated learning through self-assessment and peer assessment: Guidelines for classroom implementation. En D. Laveault y L. Allal (Eds.), Assessment for Learning: Meeting the Challenge of Implementation. The Enabling Power of Assessment (pp. 311-326). Springer. https://doi. org/10.1007/978-3-319-39211-0 18

Ruiz, J., Bados, A., Fusté, A., GarcíaGrau, E., Saldaña, C., y Lluch, T. (2016). Aprendizaje experiencial de habilidades terapéuticas y análisis de su utilidad en función de la personalidad. Behavioral Psychology (Psicología Conductual), 24(3), 405-422. https:// www.behavioralpsycho.com/producto/ aprendizaje-experiencial-de-habilidadesterapeuticas-y-analisis-de-su-utilidaden-funcion-de-la-personalidad/

Tai, J., Ajjawi, R., Boud, D., Dawson, P., y Panadero, E. (2018). Developing evaluative judgement: Enabling students to make decisions about the quality of work. Higher Education, 76, 467-481. https://doi.org/10.1007/s10734-0170220-3 
Vacha-Haase, T., Elman, N. S., Forrest, L., Kallaugher, J., Lease, S. H., Veilleux, J. C., y Kaslow, N. J. (2019). Remediation plans for trainees with problems of professional competence. Training and Education in Professional Psychology, 13(4), 239-246. https://doi.org/10.1037/tepooo0221

Van der Molen, H. (2020). Successful Communication Skills Training for the
Health Professions. Health Professions Education, 6(1), 1-2. https://doi. org/10.1016/j.hpe.2019.08.001

Villarroel, V., y Bruna, D. (2019). ¿Evaluamos lo que realmente importa? El desafío de la evaluación auténtica en educación superior. Calidad en la Educación, 50, 492-509. $\quad$ https://doi.org/10.31619/ caledu.n50.729

\section{PERFIL ACADÉMICO Y PROFESIONAL DE LAS AUTORAS}

Nieves Valencia-Naranjo. Adscrita al Departamento de Psicología de la Facultad de Humanidades y Ciencias de la Educación de la Universidad de Jaén como profesora titular. Es Doctora por la Universidad de Granada y miembro del grupo de investigación Evaluación e Intervención Psicológica (HUM-836). Ha participado en diversas publicaciones relacionadas con la psicología clínica y la discapacidad. Uno de sus intereses actuales está dirigido hacia la innovación en la formación universitaria. https://orcid.org/0000-0001-9839-4682

E-mail:nnaranjo@ujaen.es

Ma Auxiliadora Robles-Bello. Pertenece al Departamento de Psicología de la Universidad de Jaén. Es Doctora por la Universidad de Granada y miembro del grupo de investigación Evaluación e Intervención Psicológica (HUM-836). Ha sido coordinadora de la Asociación síndrome de Down y su Centro de Atención Infantil Temprana durante 20 años. Cuenta con numerosas publicaciones en revistas científicas y libros relativos a la infancia, la adolescencia, la resiliencia y la discapacidad, actualmente interesada en la innovación docente. https://orcid. org/0000-0002-4979-5399

E-mail:marobles@ujaen.es

\section{DIRECCIÓN DE LAS AUTORAS}

Edif. C-5.

C.U. Las Lagunillas $\mathrm{s} / \mathrm{n}$

Universidad de Jaén

23071 Jaén. España

Fecha de recepción del artículo: 08/08/2021

Fecha de aceptación del artículo: 22/09/2021

Fecha de aprobación para maquetación: 08/10/2021 